\title{
Impact of ESBL-producing bacteria on patients with acute pyelonephritis: A study based on patient data from a single hospital
}

\author{
SEONG-JI PARK ${ }^{1}$, IN-BEOM JEONG ${ }^{1,2}$, WON-MIN HWANG ${ }^{1,3}$, SUNG-RO YUN $^{1,3}$ and SE-HEE YOON ${ }^{1,3}$ \\ ${ }^{1}$ Department of Internal Medicine, College of Medicine, Konyang University Myunggok Medical Research Institute; \\ ${ }^{2}$ Division of Pulmonary, Allergy and Critical Care Medicine, Konyang University Hospital; ${ }^{3}$ Division of Nephrology and \\ Department of Internal Medicine, Konyang University Hospital, Daejeon 35365, Republic of Korea
}

Received January 29, 2021; Accepted June 22, 2021

DOI: $10.3892 /$ wasj.2021.113

\begin{abstract}
The present study investigated the microbial etiologies and clinical impact of extended-spectrum $\beta$-lactamase (ESBL)-producing bacteria according to urine and blood cultures from patients with acute pyelonephritis (APN). Data of all patients (age $>15$ years) who had been hospitalized at a single hospital with APN were collected between January, 2010 and December, 2018. Overall, 882 patients with APN were included. Patients were divided into an ESBL-producing and non-ESBL-producing group. In addition, each group was further stratified in accordance with positive ESBL cultures from a urine sample (U-ESBL group and U-non-ESBL group) or from either blood or urine samples (UB-ESBL group and UB-non-ESBL group). In 651 (74\%) of these cases, bacteria were isolated from either urine or blood samples. The prevalence of ESBL-producing bacteria from either urine or blood samples (UB-ESBL) was found to steadily increase over the 9-year study period from 6.3 to $37.8 \%$. The male sex [odds ratio (OR), 2.572; $95 \%$ confidence interval $(\mathrm{CI}), 1.295-5.108]$ and previous antibiotic exposure (OR, 2.275; 95\% CI, 1.105-4.680) were significant risk factors for UB-ESBL in the multivariable analysis. The patients in the UB-ESBL group exhibited a significantly higher mortality rate (OR, 1.654; 95\% CI, 1.106-2.474) and a higher continuous renal replacement therapy (CRRT) rate. By multivariable analysis, UB-ESBL and not U-ESBL was an independent prognostic indicator for mortality and recurrence. Consequently, the use of carbapenems as a first-line therapy for APN is expected to rapidly increase, and there is concern regarding the
\end{abstract}

Correspondence to: Dr Se-Hee Yoon, Division of Nephrology and Department of Internal Medicine, Konyang University Hospital, 158 Gwanjeo-dong-ro, Seo-gu, Daejeon 35365, Republic of Korea E-mail: sehei@hanmail.net

Key words: acute pyelonephritis, extended-spectrum $\beta$-lactamase, prognostic indicator, blood culture, urine culture emergence of new antibiotic-resistance bacteria. Global efforts to reduce resistance bacteria and the development of novel treatment methods other than the use of antibiotics to treat bacteria are urgently required. In addition, the results of the present study emphasize the importance of blood culture tests, as well as urine culture tests for the successful treatment of APN.

\section{Introduction}

Urinary tract infections (UTIs) are among the most common infectious diseases and include asymptomatic bacteriuria, cystitis, prostatitis and pyelonephritis (1). Among these disorders, acute pyelonephritis (APN) is a severe form of UTI. Epidemiologically, APN has an incidence of 35.7 per 10,000 individuals, among which 9.96 women and 1.18 men are hospitalized (2). APN can progress to a more severe condition, such as sepsis, septic shock and even death, and it is therefore crucial to administer appropriate antibiotics on the basis of susceptibility results (3-5).

As antibiotic resistance has gradually increased among community-acquired cases of UTIs, it has recently become more commonplace to observe an initial empirical antibiotic treatment failure in APN cases. According to the Infectious Disease Society of America (IDSA) guidelines for the treatment of UTIs, it is difficult to use an antibiotic as a primary agent when the local antibiotic resistance rate is $>20 \%$ (6). In Korea, 3rd generation cephalosporins and quinolones are commonly used for the treatment of APN as first-choice antibiotics. However, as extended-spectrum $\beta$-lactamase (ESBL)-producing bacteria have rapidly increased in frequency, it is now questionable whether they can continue to be used as first-choice interventions. In addition, blood culture tests are generally performed during the treatment of patients with APN with urine culture tests; however, the usefulness of blood cultures in APN cases remains obscure. The present study investigated the microbial etiologies and clinical impact of ESBL-producing bacteria in a series of patients with APN in order to evaluate which antibiotics are most suitable as an initial treatment for this condition. Furthermore, the necessity of blood culture tests in APN cases was analyzed. 


\section{Patients and methods}

Study design and patients. The present study performed a retrospective chart review of all patients (age $>15$ years) who had been hospitalized at Konyang University Hospital with APN between January, 2010 and December, 2018. Patients who were discharged voluntarily within 3 days and cases of transfer to other medical centers were excluded. The patients included in the study were divided into an ESBL-producing and non-ESBL producing group. In addition, each group was further stratified in accordance with positive ESBL cultures from a urine sample (U-ESBL group and U-non-ESBL group) or from either blood or urine samples (UB-ESBL group and UB-non-ESBL group) (Fig. 1). A total of two blood cultures from different sites and a urine culture were routinely conducted for each patient at the time of admission prior to antibiotic administration. The present study was approved by the Konyang Hospital Institutional Review Board (IRB approval nos. KYUH 2016-12-003-009 and KYUH 2020-06-021).

Data collection and definitions. Clinical and microbial data were collected from medical records retrospectively for all eligible patients. APN was defined by a fever $\geq 38.0^{\circ} \mathrm{C}$; at least one of the following symptoms: Flank pain, urinary frequency, urgency, dysuria and flank tenderness; and pyuria ( $\geq 10$ white blood cells per high-power field), or bacteriuria [confirmation of the isolation of a single uropathogen with $\geq 10^{5}$ colony forming units $(\mathrm{CFU}) / \mathrm{ml}$ of urine] (7). The following information was collected for the patients in the study: Age, sex, residence (i.e., rural, urban or nursing home), history of antibiotic use, comorbidities and the susceptibility of microbial isolates from blood and urine to antibiotics. Any comorbid conditions were categorized into the following disease groups: Urinary tract abnormalities (urolithiasis, Foley catheter indwelling and neurogenic bladder), cardiac disease (myocardial infarction and congestive heart failure), cerebral disease (cerebrovascular accident, dementia and hemiplegia due to brain injury) and immunocompromised conditions (kidney transplantation, chemotherapy within the prior 6 months, leukemia, lymphoma and metastatic cancer). Clinical symptoms, laboratory tests and physical manifestations were also recorded from the database. Acute Physiology and Chronic Health Evaluation (APACHE) II scores (8) were measured and recorded in patients treated in the intensive care unit (ICU). The estimated glomerular filtration rate (eGFR) was calculated using an abbreviated Modification of Diet in Renal Disease (MDRD) equation (9). The variables used for estimating treatment outcomes were the length of hospital and ICU stay, septic shock, receiving continuous renal replacement therapy (CRRT), days of antibiotic therapy, days of intravenous antibiotic therapy, a recurrence of APN within 12 months and death. Treatment failure was defined as death or the recurrence of APN.

Microbiology and susceptibility tests. For urine cultures, urine was inoculated onto 5\% sheep's blood and MacConkey agar (both from Asan Pharmaceutical Co., Ltd.) and incubated at $35^{\circ} \mathrm{C}$. The broth was maintained under these conditions for 5 days and examined for growth daily. Blood cultures was generated by inoculating $10 \mathrm{ml}$ of sampled blood into aerobic and anaerobic BD BACTEC ${ }^{\text {TM }}$ Plus (BD Diagnostics) in the appropriate medium bottles. Both aerobic and anaerobic bottles were incubated in the BD BACTEC FX blood culture system (BD Diagnostics) at $35^{\circ} \mathrm{C}$ in $5 \% \mathrm{CO}_{2}$ for 5 days. The broth was then sub-cultured in 5\% sheep's blood and chocolate agar (Asan Pharmaceutical Co., Ltd.) and incubated at $35^{\circ} \mathrm{C}$ in $5 \% \mathrm{CO}_{2}$ for 2 days. The isolated organisms were identified using the MALDI Biotyper ${ }^{\circledR}$ system (Bruker). Antimicrobial susceptibility tests were conducted using an automated MicroScan ${ }^{\circledR}$ system (Beckman Coulter, Inc.). The determination of the MIC breakpoint for each antimicrobial agent and confirmation of ESBL production were performed in accordance with the guidelines of the Clinical and Laboratory Standards Institute (CLSI) (10).

Statistical analysis. Demographic and clinical characteristics were presented as percentages for categorical variables and as the mean \pm standard deviation (SD) for continuous variables. Chi-squared tests were used to compare categorical variables and Student t-tests were used for continuous variables. The Chi-squared test or Fisher's exact test were used to analyze the changes in antibiotic susceptibility and percentage of isolates producing ESBLs. Logistic regression analysis was used to estimate the odds ratios (ORs) with $95 \%$ confidence intervals (CIs). Variables with a P-value of $<0.05$ in the unadjusted models were entered into the multivariable regression model. Cox proportional hazards regression models were used to investigate the impact of ESBL on treatment outcomes. To further adjust for potential confounders, inverse probability weights were incorporated into the Cox proportional hazard regression models. The weights for the ESBL group were the inverse of the propensity scores, and those for the non-ESBL group were the inverse of 1-the propensity scores (11). Variables that were considered potential confounders based on previous literature (11-17) or clinical relevance to the model, i.e., age, sex, Foley catheter indwelling, immunocompromised condition and nursing home residence were included. All statistical analyses were performed using R 4.0.0 (www.r-project. org). P-values $<0.05$ were considered to indicate statistically significant differences.

\section{Results}

Study population and isolated pathogens. A total of 882 patients were hospitalized with APN at Konyang University Hospital from January, 2010 to December, 2018 and were enrolled in the initial study population. In $580(65.7 \%)$ of these cases, bacteria were cultured from urine samples. Otherwise, in $651(73.8 \%)$ of the APN cases, bacteria were cultured from either urine or blood samples. The isolated bacteria and their frequency in the study population are presented in Table I. Notably, in 71 (8.0\%) patients, no bacteria grew in the urine cultures, but were detectable in the blood cultures. The discordance rate for the bacteria isolated from blood and urine cultures was $4.3 \%$.

Antibacterial susceptibility. Escherichia coli (E. coli) was found to be the most common cause of APN in the present study cohort, exhibiting a sensitivity of $29.1 \%$ to ampicillin 
Table I. Bacterial isolates in the urine and blood cultures of patients with acute pyelonephritis.

\begin{tabular}{|c|c|c|c|}
\hline Bacterial isolates & Urine culture & Blood culture & Either urine or blood culture \\
\hline Escherichia coli, no. (\%) & $505(57.4)$ & $267(30.3)$ & $522(59.2)$ \\
\hline Klebsiella Spp, no. (\%) & $23(2.6)$ & $8(0.9)$ & $18(2)$ \\
\hline Enterococcus Spp, no. (\%) & $16(1.8)$ & $11(1.2)$ & $18(2)$ \\
\hline Proteus Spp, no. (\%) & $8(0.9)$ & $2(0.2)$ & $7(0.8)$ \\
\hline Enterobacter Spp, no. (\%) & $6(0.7)$ & $3(0.3)$ & $5(0.6)$ \\
\hline Pseudomonas Spp, no. (\%) & $5(0.6)$ & $2(0.2)$ & $3(0.3)$ \\
\hline Candida Spp, no. (\%) & $3(0.3)$ & 0 & $2(0.2)$ \\
\hline Two more Spp, no. (\%) & & $6(0.7)$ & $44(5)$ \\
\hline Other Spp, no. $(\%)$ & $14(1.6)$ & $47(5.3)$ & $32(3.6)$ \\
\hline Total isolates, no. (\%) & $580(65.8)$ & $346(39.2)$ & $651(73.8)$ \\
\hline No culture test, no. (\%) & $4(0.5)$ & $2(0.2)$ & $2(0.2)$ \\
\hline No growth, no. (\%) & $298(33.8)$ & $534(60.5)$ & $229(26)$ \\
\hline
\end{tabular}

All percentages were calculated out of the total number of included patients $(n=882)$. Spp; species, no., number.

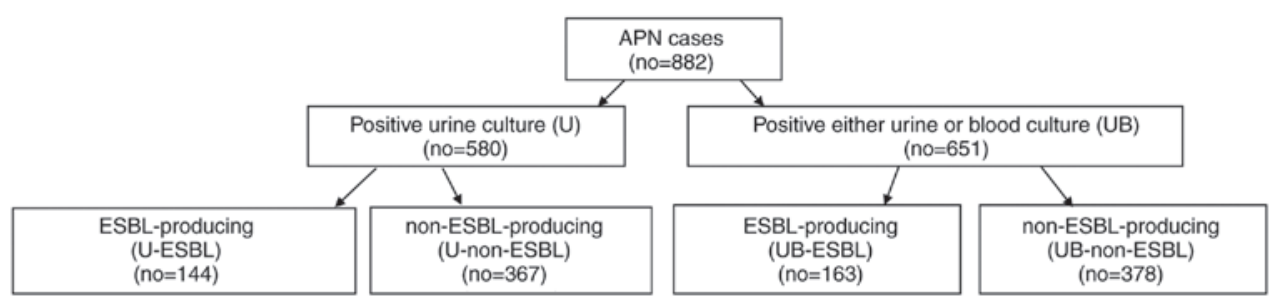

Figure 1. Flow chart demonstrating patient stratification into culture tests and ESBL production. APN, acute pyelonephritis; no, number; ESBL, extended spectrum $\beta$-lactamase; $\mathrm{U}$, urine culture; $\mathrm{UB}$, either urine or blood culture.

and $36.6 \%$ to ampicillin/sulbactam. The sensitivity level to ceftazidime, cefepime and ciprofloxacin was $>70 \%$, but $<80 \%$. However, the sensitivity to piperacillin/tazobactan, meropenem and amikacin was $>90 \%$. ESBL-producing $E$. coli were $27.2 \%$. More details on the susceptibility test results for isolated bacteria other than $E$. coli, such as Klebsiella, Enterococcus, Proteus, Enterobacter and Pseudomonas are listed in Table SI. The changes in the isolated uropathogen antibiotic susceptibility levels over the 9-year study period from 2010 to 2018 are illustrated in Fig. 2. The bacterial susceptibility to ampicillin (28.4\%), ampicillin/sulbactam (38.9\%), piperacillin/tazobactam $(95.4 \%)$, ciprofloxacin $(70.2 \%)$ and amikacin $(97.5 \%)$ had not changed significantly over this time period $(\mathrm{P}=0.802$, $0.960,0.258,0.091$ and 0.747 , respectively). However, the susceptibility rate to ampicillin and ampicillin/sulbactam remained $<40 \%$ during this time period. Notably, the bacterial susceptibility to ceftazidime and cefepime significantly decreased during the observation period from $90 \%$ on average in 2010 to $70 \%$ on average in $2018(\mathrm{P}=0.003$ and 0.039 , respectively). The percentage of ESBL-producing bacteria detected over the 9 -year study period is shown in Fig. 3. This was $6.3 \%$ in 2010 , but had markedly increased to $>30 \%$ after $2015(\mathrm{P}=0.001)$.

Risk factors for ESBL acquisition. The baseline characteristics of the patients in the present study are demonstrated with the univariate analysis in Table SII. Age, male sex and a rural area or nursing home residence were found to be significantly associated with both the U- and UB-ESBL groups. As regards comorbid conditions, Foley catheter indwelling, a cerebrovascular accident and dementia were also significantly associated with both groups. Of note, a history of antibiotic treatment was found to be significantly associated with only the UB-ESBL group. By multivariate analysis, it was found that the male sex was an independent risk factor for $\mathrm{U}-\mathrm{ESBL}(\mathrm{OR}, 2.457 ; \mathrm{P}=0.015)$. On the other hand, the male sex and a history of antibiotic treatments were identified in this analysis as independent risk factors for UB-ESBL (OR, 2.572; $\mathrm{P}=0.007$; $\mathrm{OR}, 2.275 ; \mathrm{P}=0.026$, respectively) (Table II).

Clinical features of the ESBL and non-ESBL groups. The severity of illness of the study patients is presented in Table III. When the severity of illness in the U-ESBL group and UB-ESBL group was analyzed, respectively, the mean body temperature and febrile period differed between the ESBL and non ESBL groups. The other variables did not exhibit any significant differences between groups.

Treatment and clinical outcomes in the ESBL group. As regards the final treatment outcomes, there were nine treatment failures, including four clinical recurrences and five deaths. In the unadjusted analysis, the patients in the 


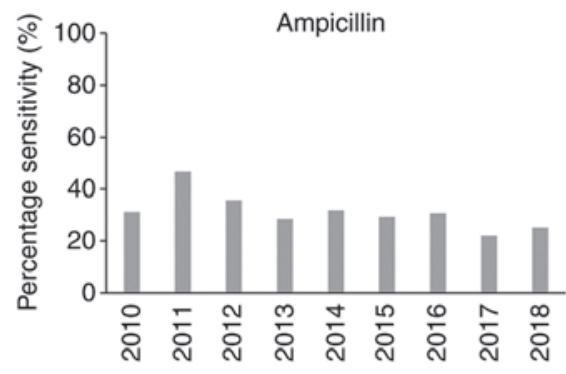

Piperacillin/Tazobactam

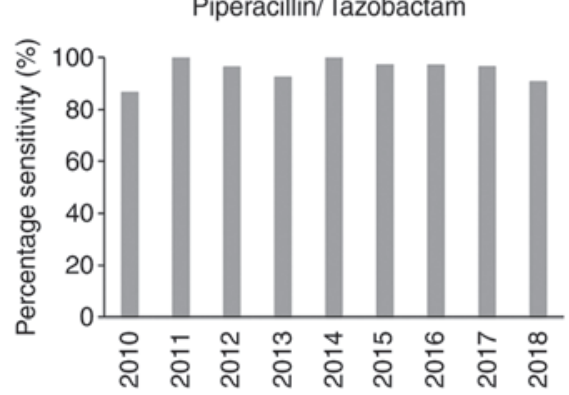

Amikacin

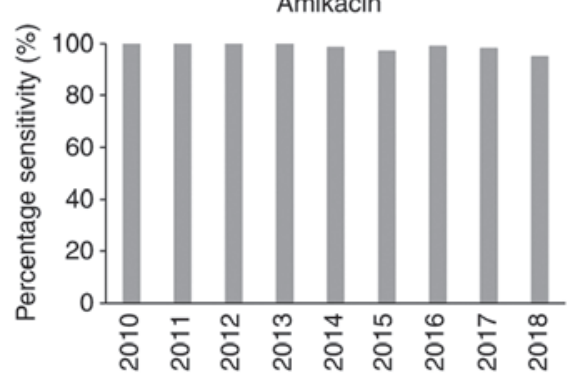

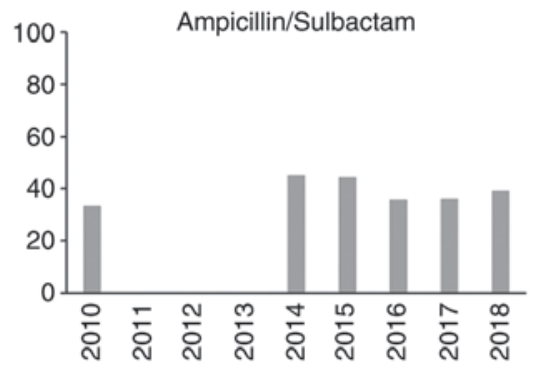

Ceftazidime

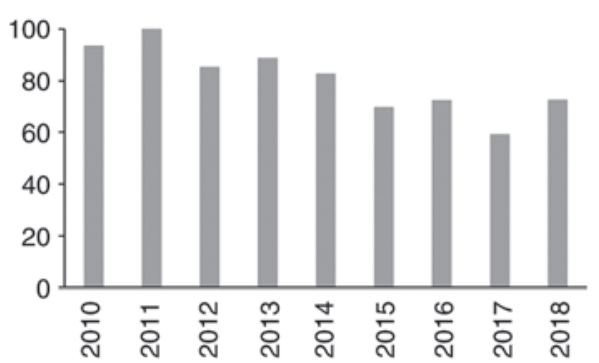

Meropenem

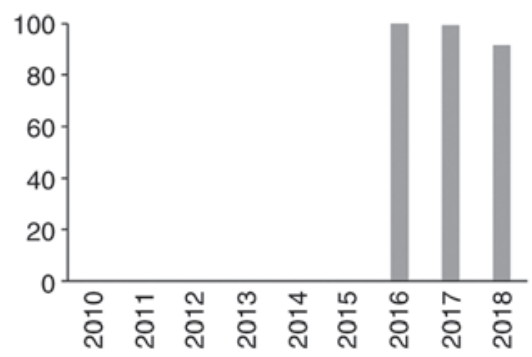

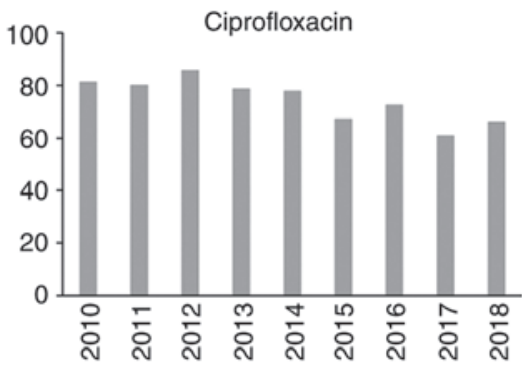

Cefepime

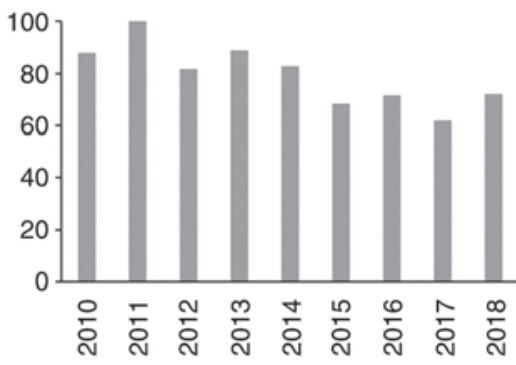

Figure 2. Antibiotic susceptibility of bacterial isolates from patients with acute pyelonephritis over the 9-year period.

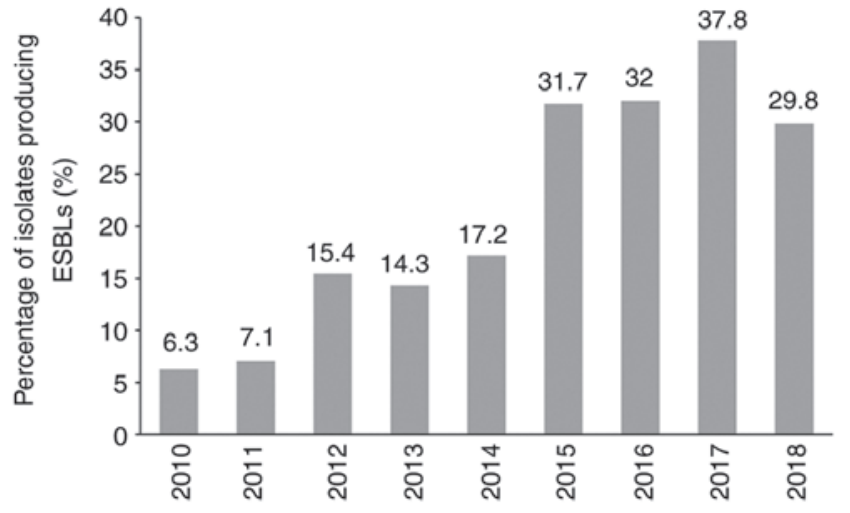

Figure 3. Changes in the incidence of ESBL-producing bacteria in acute pyelonephritis patients over time. ESBL, extended spectrum $\beta$-lactamase.

UB-ESBL group exhibited a significantly higher mortality rate and a higher CRRT rate than the non-UB-ESBL (negative) cases (OR, 1.654; $\mathrm{P}=0.015 ; \mathrm{OR}, 1.447 ; \mathrm{P}=0.049$, respectively). However, the rate of clinical recurrences did not differ significantly different between these groups (OR, 1.222; $\mathrm{P}=0.386)$. The duration of intravenous antibiotic therapy and the length of hospital and ICU stay were significantly higher in the UB-ESBL group (Table IV). Similar results were observed between the UB-ESBL and U-ESBL groups. The results of treatment and outcomes in the patients with APN according to U-ESBL are presented in Table SIII.

Prognostic factors for APN treatment outcomes. UB-ESBL was found to be an independent prognostic factor along with septic shock for poor outcomes of treatment for APN [hazard ratio (HR), 8.380 $\mathrm{P}=0.0002$; $\mathrm{HR}, 4.538 ; \mathrm{P}=0.0252$, respectively]. To further control for potential confounding, a pseudo-population was created by applying weights so that the demographic and baseline characteristics were well balanced between the groups. When weights were incorporated into a multivariable regression model, UB-ESBL was the only independent prognostic factor to emerge (HR, 8.439, $\mathrm{P}=0.0008$ ) (Table V). A multivariable analysis of U-ESBL cases was also performed and it was found that this variable did not negatively affect clinical outcomes. The independent prognostic factor for adverse outcomes from APN was septic shock. When weights were incorporated into a multivariable regression model, U-ESBL was not associated with clinical failure (HR, 2.710; $\mathrm{P}=0.915$ ) (Table SIV).

\section{Discussion}

The present study investigated the microbial etiologies and clinical impact of ESBL-producing bacteria in a series of patients with APN over a 9-year period. The prevalence of ESBL gradually increased and exceeded 30\% from 2015. 
Table II. Multivariate analysis for risk factors associated with infection by ESBL-producing isolates that cause acute pyelonephritis.

\begin{tabular}{|c|c|c|c|c|}
\hline \multirow[b]{2}{*}{ Variable } & \multicolumn{2}{|c|}{ U-ESBL } & \multicolumn{2}{|c|}{ UB-ESBL } \\
\hline & OR $(95 \% \mathrm{CI})$ & P-value & OR $(95 \% \mathrm{CI})$ & P-value \\
\hline Age, $>65$ years & $1.095(0.705-1.699)$ & 0.686 & $1.098(0.720-1.673)$ & 0.664 \\
\hline Sex, male & $2.457(1.192-5.067)$ & 0.015 & $2.572(1.295-5.108)$ & 0.007 \\
\hline Residence, rural area & $1.712(0.950-3.085)$ & 0.073 & $1.712(0.991-2.956)$ & 0.054 \\
\hline Residence, nursing home & $1.632(0.740-3.595)$ & 0.225 & $2.056(0.974-4.343)$ & 0.059 \\
\hline Foley catheter & $3.725(0.895-15.509)$ & 0.071 & $1.157(0.357-3.749)$ & 0.808 \\
\hline Cerebral disease $^{\mathrm{a}}$ & $1.823(0.980-3.390)$ & 0.058 & $1.566(0.860-2.852)$ & 0.142 \\
\hline Previous antibiotic exposure & $1.731(0.772-3.879)$ & 0.183 & $2.275(1.105-4.680)$ & 0.026 \\
\hline
\end{tabular}

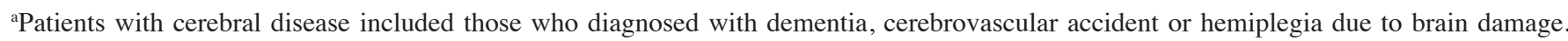
ESBL, extended spectrum $\beta$-lactamase; U, urine culture; UB, either urine or blood culture; OR, odds ratio; CI, confidence interval.

Table III. Clinical features of patients with acute pyelonephritis according to the presence of ESBL-producing bacteria.

\begin{tabular}{|c|c|c|c|c|c|c|}
\hline \multirow[b]{2}{*}{ Variable } & \multicolumn{3}{|c|}{ U-ESBL } & \multicolumn{3}{|c|}{ UB-ESBL } \\
\hline & $\begin{array}{l}\text { Positive } \\
(n=144)\end{array}$ & $\begin{array}{l}\text { Negative } \\
(n=367)\end{array}$ & P-value & $\begin{array}{l}\text { Positive } \\
(n=163)\end{array}$ & $\begin{array}{l}\text { Negative } \\
(n=378)\end{array}$ & P-value \\
\hline APACHE II score, mean (SD) & $15.36(5.085)$ & $12.60(2.757)$ & 0.137 & $16.46(6.86)$ & $13.08(3.90)$ & 0.148 \\
\hline Temperature $\left({ }^{\circ} \mathrm{C}\right)$, mean $(\mathrm{SD})$ & $37.49(0.940$ & $37.72(1.063)$ & 0.019 & $37.47(0.93)$ & $37.71(1.06)$ & 0.013 \\
\hline Mean BP (mmHg), mean (SD) & $82.5(10.621)$ & $83.61(12.151)$ & 0.337 & $82.51(10.50)$ & $83.60(12.09)$ & 0.319 \\
\hline PR (beats/min), mean (SD) & $84.85(14.088)$ & $83.85(15.516)$ & 0.502 & $85.23(13.98)$ & $83.73(15.45)$ & 0.287 \\
\hline RR (breaths/min), mean (SD) & $20.03(2.046)$ & $20.36(3.225)$ & 0.258 & $20.12(2.07)$ & $20.37(3.19)$ & 0.357 \\
\hline eGFR $\left(\mathrm{ml} / \mathrm{min} / 1.73 \mathrm{~m}^{2}\right)$, mean $(\mathrm{SD})$ & $67.44(33.06)$ & $72.78(32.85)$ & 0.122 & $70.42(37.05)$ & $72.58(32.87)$ & 0.526 \\
\hline WBC $\left(10^{3} / \mu 1\right)$, mean $(\mathrm{SD})$ & $13.61(5.671)$ & $12.74(5.493)$ & 0.110 & $13,780(5808)$ & $12,790(5,477)$ & 0.065 \\
\hline CRP (mg/dl), mean (SD) & $14.74(9.568)$ & $13.30(8.947)$ & 0.112 & $15.24(9.58)$ & $13.55(9.11)$ & 0.071 \\
\hline Total $\mathrm{CO}_{2}(\mathrm{mmol} / \mathrm{l})$, mean $(\mathrm{SD})$ & $21.34(4.079)$ & $21.84(3.577)$ & 0.319 & $21.28(4.00)$ & $21.90(3.63)$ & 0.184 \\
\hline Febrile period (days), mean (SD) & $3.20(2.986)$ & $2.28(1.651)$ & 0.001 & $3.29(3.156)$ & $2.29(1.734)$ & 0.001 \\
\hline
\end{tabular}

ESBL, extended-spectrum $\beta$-lactamase; APACHE, Acute Physiology and Chronic Health Evaluation; BP, blood pressure; PR, pulse rate; $\mathrm{BR}$, respiratory rate; eGFR, estimated glomerular filtration rate; WBC, white blood cell; CRP, C reactive protein; U, urine; UB, either urine or blood.

UB-ESBL, but not U-ESBL, was found to be an independent prognostic factor of poor clinical outcomes along with septic shock. Significant risk factors for UB-ESBL were the male sex and previous antibiotic exposure in the present study series.

The prevalence of ESBL-producing bacteria in UTIs has been reported in previous studies to range from 8.7 to $50.5 \%(12-17)$. In the current study, the prevalence of ESBL-producing bacteria patients with APN gradually increased, from $6.3 \%$ in 2010 to $30 \%$ in 2018 . As the antibiotic resistance of causative bacteria of APN rapidly increases, recently updated 2018 Korean guidelines recommended for early empirical antibiotic administration for patients with APN who require hospitalization, broad spectrum antibiotics such as 3rd generation cephalosporins, piperacillin-tazobacam, or carbapenems until susceptibility results are obtained (18). As the guidelines did not recommend only one specific antibiotic, they offer many options depending on the situation, the choice of empiric antibiotics for APN patients should be made by the physicians based on the regional frequency of antibiotic resistance and the severity of illness. Based on the results of the present study, the use of carbapenem as a primary treatment for APN in Korea is expected to increase rapidly. The development of carbapenem resistant bacteria is of concern. The resistance of the isolated bacteria to meropenem in the patients with APN in the present study gradually increased over the study period, although the susceptibility testing for carbapenem was performed from 2016.

The multivariate analysis identified a male sex and previous antibiotic exposure as risk factors for ESBL-producing bacterial infection. Previous studies have reported varied risk factors for 
Table IV. Treatment of acute pyelonephritis according to the presence of ESBL-producing bacteria.

UB-ESBL

\begin{tabular}{lcccr}
\cline { 2 - 4 } Variables & Positive (n=163) & Negative (n=378) & OR (95\% CI) & P-value \\
\hline Hospital stay (days), mean (SD) & $14.31(9.71)$ & $8.01(4.26)$ & & 0.001 \\
ICU stay (days), mean (SD) & $0.80(2.33)$ & $0.32(1.168)$ & & 0.013 \\
Septic shock, no. (\%) & $11(6.0)$ & $21(5.6)$ & $1.046(0.888-1.233)$ & 0.59 \\
CRRT, no. (\%) & $4(2.0)$ & $2(0.5)$ & $1.447(1.001-2.092)$ & 0.049 \\
Days of antibiotic therapy, mean (SD) & $14.83(8.04)$ & $14.48(5.416)$ & & 0.609 \\
Days of IV antibiotic use, mean (SD) & $13.23(7.64)$ & $7.20(3.34)$ & & 0.001 \\
Recurrence, no. (\%) & $2(1.2)$ & $2(0.5)$ & $1.222(0.778-1.919)$ & 0.386 \\
Mortality, no. (\%) & $4(2.5)$ & $1(0.3)$ & $1.654(1.106-2.474)$ & 0.015
\end{tabular}

ESBL, extended-spectrum $\beta$-lactamase; ICU, intensive care unit, no number; CRRT, continuous renal replacement; therapy; IV, intravenous; UB, either urine or blood; OR, odds ratio; CI, confidence interval.

Table V. Multivariable analysis for prognostic factors associated with clinical failure in patients with acute pyelonephritis.

\begin{tabular}{|c|c|c|c|c|}
\hline \multirow[b]{2}{*}{ Variable } & \multicolumn{2}{|c|}{ Unweighted } & \multicolumn{2}{|c|}{ Weighted } \\
\hline & $\mathrm{HR}(95 \% \mathrm{CI})$ & P-value & $\mathrm{HR}(95 \% \mathrm{CI})$ & P-value \\
\hline UB-ESBL & $8.380(2.7067-25.946)$ & 0.0002 & 8.439 (2.3986-29.694) & 0.0008 \\
\hline Age, $>65$ years & $1.050(0.2914-3.785)$ & 0.9404 & $1.462(0.2115-10.111)$ & 0.7000 \\
\hline Sex, male & $1.692(0.4364-6.6561)$ & 0.4468 & $1.471(0.3273-6.613)$ & 0.6147 \\
\hline Cardiac disease $^{\mathrm{a}}$ & $1.726(0.2088-14.260)$ & 0.6126 & $4.900(0.5790-41.479)$ & 0.1447 \\
\hline Cerebral disease $^{\mathrm{b}}$ & $1.470(0.3736-5.785)$ & 0.5814 & $1.842(0.3655-9.288)$ & 0.4590 \\
\hline Septic shock & $4.538(1.2070-17.064)$ & 0.0252 & $4.450(0.9338-21.209)$ & 0.0609 \\
\hline
\end{tabular}

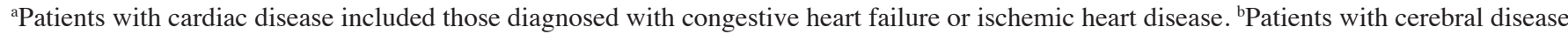
patients included cases of dementia, cerebrovascular accident or hemiplegia due to brain damage. ESBL, extended-spectrum $\beta$-lactamase; $\mathrm{UB}$, either urine or blood; HR, hazard ratio; CI, confidence interval.

ESBL-producing bacteria, and such as male or female sex, cerebrovascular disease, intubation/tracheostomy, major surgery within 60 days, immunocompromised status and antimicrobial usage during the preceding 60 days $(11,19)$. The present study has confirmed many of the findings of these previous studies.

Some previous studies have reported a higher mortality rate for patients infected with ESBL-producing bacteria (20-23), whereas other reports have not noted this difference (24). In the present study, it was found that the UB-ESBL variable played an important role in predicting disease mortality and recurrences corresponding to clinical failure. The present study further subdivided the ESBL-group and non-ESBL groups into positive/negative urine culture (U-ESBL, non-U-ESBL) and either urine or blood culture (UB-ESBL, non-UB-ESBL) groups. The results indicated that both urine and blood culture tests are important for the proper treatment of APN patients. In the present study, the blood cultures were positive in 71 patients whose bacteria were not cultured in the urine culture. In 19 of these patients, ESBL-producing bacteria were identified in a blood culture. Velasco et al (25) suggested that there was no need to perform a blood culture test in patients with APN due to the low discordance rate and no difference in the clinical course of patients whose urine culture and blood culture results do not match. Antibiotic susceptibility testing is usually not performed in the laboratory if the patient's bacterial count is $<10^{3} \mathrm{CFU}$. However, if the patient is exposed to antibiotics prior to the visit to the hospital or the urine is diluted, the bacteria in the urine sample may not grow to $10^{3} \mathrm{CFU}$ and a positive case of UTI may go undetected. In these cases, as shown in the present study, blood culture tests play an important role in conjunction with urine culture tests for detecting APN and initiating the appropriate treatment.

The present study had several limitations which should be noted. In the first instance, this was a retrospective chart review study conducted at a single center which can have introduced selection and information biases. Bacteria were not identified from cultures in $33.8 \%$ of the current cases and the treatments of these patients were therefore not based on the causative microbes. There was also no information on the bacterial genotypes. Since there were very few patients with recurrence within 6 months, patients with recurrence 
of APN within 1 year were included in the treatment failure. Notwithstanding these limitations however, the strength of the present study was that recent data from a large cohort of 882 patients that had been treated for APN over the past 9 years were analyzed. The authors also tried to overcome the inherent limitations of a retrospective chart review study by incorporating inverse probability weights when analyzing the impact of ESBL-producing bacteria on clinical outcomes.

In conclusion, the present study demonstrates that the frequency of ESBL-producing bacteria in APN cases now exceeds $30 \%$ and UB-ESBL was associated with poor clinical outcome. The use of carbapenems is expected to increase, and there is concern regarding the occurrence of resistance bacteria. Accordingly, global efforts to reduce resistance bacteria and the development of new treatment method to treat bacteria are required. In addition, the results of the present study have emphasized the importance of blood culture test, as well as urine culture test for the successful treatment of APN.

\section{Acknowledgements}

Not applicable.

\section{Funding}

The present study was supported by the Basic Science Research Program of the National Research Foundation of Korea (NRF), funded by the Ministry of Education (grant no. NRF-2020R1I1A3A04037055 and NRF-2017R1A6A1A03015713).

\section{Availability of data and materials}

The datasets generated and/or analyzed during the current study are not publicly available due to the confidentiality of clinical data, but are available from the corresponding author on reasonable request.

\section{Authors' contributions}

SJP, IBJ, WMH, SRY and SHY conceived, performed data analysis and designed the experiments. SJP and SHY drafted the manuscript and finalized the manuscript. SJP and SHY confirm the authenticity of all the raw data. All authors have read and approved the final manuscript.

\section{Ethics approval and consent to participate}

The present study was reviewed and approved by the institutional review board of Konyang University Hospital. (IRB approval no. KYUH 2016-12-003-009 and KYUH 2020-06-021).

\section{Patient consent for publication}

Not applicable.

\section{Competing interests}

The authors declare that they have no competing interests.

\section{References}

1. Najar MS, Saldanha CL and Banday KA: Approach to urinary tract infections. Indian J Nephrol 19: 129-139, 2009.

2. Ki MR, Park TS, Choi BY and Foxman B: The epidemiology of acute pyelonephritis in South Korea, 1997-1999. Am J Epidemiol 160: 985-993, 2004.

3. Johnson JR and Russo TA: Acute pyelonephritis in adults. N Engl J Med 378: 48-59, 2018.

4. Gupta K, Hooton TM, Naber KG, Wullt B, Colgan R, Miller LG, Moran GJ, Nicolle LE, Raz R, Schaeffer AJ, et al: International clinical practice guidelines for the treatment of acute uncomplicated cystitis and pyelonephritis in women: A 2010 update by the Infectious Diseases society of America and the European society for microbiology and infectious diseases. Clin Infect Dis 52: e103-e120, 2011.

5. Pietrucha-Dilanchian P and Hooton TM: Diagnosis, treatment, and prevention of urinary tract infection. Microbiol Spectr: doi: 10.1128/microbiolspec.UTI-0021-2015.

6. Warren JW, Abrutyn E, Hebel JR, Johnson JR, Schaeffer AJ and Stamm WE: Guidelines for antimicrobial treatment of uncomplicated acute bacterial cystitis and acute pyelonephritis in women. Infectious diseases society of America (IDSA) Clin Infect Dis 29: 745-758, 1999.

7. Bosch-Nicolau P, Falcó V, Viñado B, Andreu A, Len O, Almirante B and Pigrau C: Risk factors that influence the empirical treatment of patients with acute pyelonephritis: A cohort study. Antimicrob Agents Chemother 61: AAC.01317-17, 2017.

8. Knaus WA, Draper EA, Wagner DP and Zimmerman JE: APACHE II: A severity of disease classification system. Crit Care Med 13: 818-829, 1985.

9. Levey AS, Coresh J, Greene T, Stevens LA, Zhang YL, Hendriksen S, Kusek JW and Lente FV: Chronic Kidney Disease Epidemiology Collaboration: Using standardized serum creatinine values in the modification of diet in renal disease study equation for estimating glomerular filtration rate. Ann Intern Med 145: 247-254, 2006.

10. Clinical and Laboratory Standards Institute (CLSI): Performance standards for antimicrobial susceptibility testing; 17th informational supplement. Approved standard M100-S17. CLSI, Annapolis Junction, MD, 2007.

11. Park SH, Choi SM, Lee DG, Cho SY, Lee HJ, Choi JK, Choi JH and Yoo JH: Impact of extended-spectrum $\beta$-lactamase production on treatment outcomes of acute pyelonephritis caused by Escherichia coli in patients without health care-associated risk factors. Antimicrob Agents Chemother 59: 1962-1968, 2015.

12. Tüzün T, Sayın Kutlu S, Kutlu M and Kaleli İ: Risk factors for community-onset urinary tract infections caused by extended-spectrum $\beta$-lactamase-producing Escherichia coli. Turk J Med Sci 49: 1206-1211, 2019.

13. Albaramki JH, Abdelghani T, Dalaeen A, Khdair Ahmad F, Alassaf A, Odeh R and Akl K: Urinary tract infection caused by extended-spectrum $\beta$-lactamase-producing bacteria: Risk factors and antibiotic resistance. Pediatr Int 61: 1127-1132, 2019.

14. Kim B, Kim J, Seo MR, Wie SH, Cho YK, Lim SK, Lee JS, Kwon KT, Lee H, Cheong HJ, et al: Clinical characteristics of community-acquired acute pyelonephritis caused by ESBL-producing pathogens in South Korea. Infection 41: 603-612, 2013.

15. Díaz-Agero Pérez C, López-Fresneña N, Rincon Carlavilla AL, Hernandez Garcia M, Ruiz-Garbajosa P, Aranaz-Andrés JM, Maechler F, Gastmeier P, Bonten MJM and Canton R: Local prevalence of extended-spectrum beta-lactamase (ESBL) producing enterobacteriaceae intestinal carriers at admission and co-expression of ESBL and OXA-48 carbapenemase in Klebsiella pneumoniae: A prevalence survey in a Spanish university hospital. BMJ Open 9: e024879, 2019.

16. Karanika S, Karantanos T, Arvanitis M, Grigoras C and Mylonakis E: Fecal colonization with extended-spectrum beta-lactamase-producing enterobacteriaceae and risk factors among healthy individuals: A systemic review and metaanalysis. Clin Infect Dis 63; 310-318, 2016.

17. Kim YJ, Lee JM, Cho J and Lee JH: Change in the annual antibiotic susceptibility of Escherichia coli in community-onset urinary tract infection between 2008 and 2017 in a tertiary care hospital in Korea. J Korean Med Sci 34: e228, 2019. 
18. Kang CI, Kim J, Park DW, Kim BN, Ha US, Lee SJ, Yeo JK, Min SK, Lee H and Wie SH: Clinical practice guidelines for the antibiotic treatment of community-acquired urinary tract infections. Infect Chemother 50: 67-100, 2018

19. Luvsansharav UO, Hirai I, Nakata A, Imura K, Yamauchi $K$, Niki M, Komalamisra C, Kusolsuk T and Yamamoto Y: Prevalence of and risk factors associated with faecal carriage of CTX-M $\beta$-lactamase-producing enterobacteriaceae in rural Thai communities. J Antimicrob Chemother 67: 1769-1774, 2012.

20. Schwaber MJ and Carmeli Y: Mortality and delay in effective therapy associated with extended-spectrum beta-lactamase production in enterobacteriaceae bacteraemia: A systematic review and meta-analysis. J Antimicrob Chemother 60: 913-920, 2007.

21. Marchaim D, Gottesman T, Schwartz O, Korem M, Maor Y, Rahav G, Karplus R, Lazarovitch T, Braun E, Sprecher H, et al: National multicenter study of predictors and outcomes of bacteremia upon hospital admission caused by enterobacteriaceae producing extended-spectrum beta-lactamases. Antimicrob Agents Chemother 54: 5099-5104, 2010.

22. Freeman JT, McBride SJ, Nisbet MS, Gamble GD, Williamson DA, Taylor SL and Holland DJ: Bloodstream infection with extended-spectrum beta-lactamase-producing Enterobacteriaceae at a tertiary care hospital in New Zealand: Risk factors and outcomes. Int J Infect Dis 16: e371-e374, 2012.
23. Qureshi ZA, Paterson DL, Peleg AY, Adams-Haduch JM, Shutt KA, Pakstis DL, Sordillo E, Polsky B, Sandkovsky G, Bhussar MK and Doi Y: Clinical characteristics of bacteraemia caused by extended-spectrum $\beta$-lactamase-producing enterobacteriaceae in the era of CTX-M-type and KPC-type $\beta$-lactamases. Clin Microbiol Infect 18: 887-893, 2012

24. Lee NY, Lee CC, Huang WH, Tsui KC, Hsueh PR and Ko WC: Carbapenem therapy for bacteremia due to extended-spectrum$\beta$-lactamase-producing Escherichia coli or Klebsiella pneumoniae: Implications of ertapenem susceptibility. Antimicrob Agents Chemother 56: 2888-2893, 2012.

25. Velasco M, Martínez JA, Moreno-Martínez A, Horcajada JP, Ruiz J, Barranco M, Almela M, Vila J and Mensa J: Blood cultures for women with uncomplicated acute pyelonephritis: Are they necessary? Clin Infect Dis 37: 1127-1130, 2003.

This work is licensed under a Creative Commons Attribution-NonCommercial-NoDerivatives 4.0 International (CC BY-NC-ND 4.0) License. 